\title{
Impact of Covid on Mental Status of Health Care Workers and in General Population
}

\section{Avineet Kaur ${ }^{1 *}$, Shalika ${ }^{2}$, Gaganpreet Singh ${ }^{3}$, Jaskaran Singh ${ }^{4}$, Ravneet Kaur Bhangu ${ }^{5}$ and Sahil Sheokand ${ }^{6}$}

${ }^{1}$ Senior Lecturer, Department of Periodontology and Oral Implantology, Swami

Devi Dyal Dental College and Hospital Golpura, Barwala, Haryana, India

${ }^{2} J$ unior Resident, Department of Dermatology, Gian Sagar Medical Hospital, Banur,

Rajpura, Punjab, India

${ }^{3}$ Medical Officer, Civil Hospital Jalandhar, Punjab, India

${ }^{4}$ Senior Resident, Post Graduate Institute of Medical Education and Research,

Chandigarh, India

${ }^{5}$ Medical Officer, Department of Medicine, Mohandai Oswal Hospital, Ludhiana,

Punjab, India

${ }^{6} P G 3^{\text {rd }}$ Year, Department of Medicine, Maharishi Markandeshwar College of

Medical Sciences and Research Mullana Ambala, Haryana, India

*Corresponding Author: Avineet Kaur, Senior Lecturer, Department of

Periodontology and Oral Implantology, Swami Devi Dyal Dental College and Hospital

Golpura, Barwala, Haryana, India.
Received: July 19, 2021

Published: July 30, 2021

(C) All rights are reserved by Avineet Kaur., et al.

\begin{abstract}
Coronaviruses consist of enveloped viruses. The easily transmittable (COVID-19) is a viral infection originating from Wuhan, China. The unmatched general epidemic of (COVID-19) has continued to have a remarkable force on nations. Government controls and restrictions were put in place along with social isolation and distancing thus slowing down the spread of the virus.
\end{abstract}

Keywords: Coronavirus; Mental Status; Health Care Workers

\section{Introduction}

Coronaviruses (CoV) belong to the genus Coronaviridae. They are being pleomorphic in nature. Peplomers basically consist of 80-160 nM in size and 27-32 kb with positive polarity contained by RNA viruses shaped in crown fashion. Coronaviruses are zoonotic pathogens. Mutation rates are very high that exist in humans and animals causing infections related to respiration, hepatics, neurology and the gastrointestinal system [1].

In the province of Guangdong China in 2002 and 2003 for the first time a highly pathogenic virus called SARS was found in hu- mans which mainly affected the populations with weaker immunity. It has spread as CoV OC43 and CoV229E. On December 12, 2019, the first unknown case of pneumonia was detected which soon after known as novel (COVID-19) [1]. It was observed previously and research carried on infectious diseases which includes Severe Acute Respiratory Syndrome (SARS), the Middle East respiratory syndrome (MERS) and the Ebola virus disease and time and again revealed that many healthcare professionals (HPs) disclosed symptoms of depression and anxiety causing a stern force on their coping abilities. 
Above and beyond, Great Recession (2008) was followed by economic cuts which was due to reduced material and human resources was faced by public healthcare systems. The circumstances deteriorated additionally during the COVID-19 epidemic [2].

\section{Pathophysiology}

SARS-CoV-2 is the third coronavirus which is so severe in nature that it spread internationally in the precedent 2 decades. The first coronavirus is (SARS) in year 2002-2003, that derive in Foshan, China. The second was the coronavirus ( MERS), that commenced from the Arabian peninsula in 2012. SARS-CoV-2 have distinctive spikes, which ranged from $9 \mathrm{~nm}$ to $12 \mathrm{~nm}$ and a diameter of $60 \mathrm{~nm}$ to $140 \mathrm{~nm}$ and distinctive spikes, displaying the virions the resembling the solar corona. Humans became infected with SARSCoV-2 via host pangolin although bat is being a natural reservoir [3].

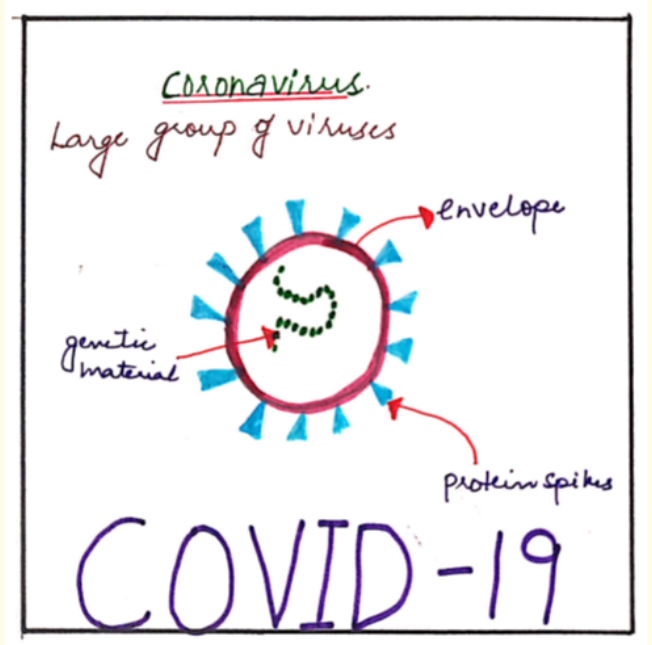

Figure 1

\section{Clinical Presentation}

Incubation period for COVID-19 is something like 5 (2 - 7) days. Out which $97.5 \%$ symptoms within 11.5 days of infection. Fourty seven to seventy three years are being hospitalized, with mostly males. Symptoms are fever in $90 \%$ cases, cough in $60 \%-86 \%$ cases and shortness of breath in 53\%-80\% cases. Patients can also be in attendance with non conventional symptoms, such as Olfactory, gastrointestinal and loss of gustatory function have been depicted in $64 \%$ to $80 \%$ of patients.

\section{Impact of COVID 19 on children and teens at risk [4,5]}

Children when absent from school they may have many questions about the outbreak and they expect the answer from parents. Although many parents are not able to deal with stress. Kids and teens experience social distancing, anxiety and abusive environment.

Crying and annoying behavior, sadness and depression. Hence difficulties in concentrating and paying attention. Changes in appetite unexpected headaches and pain. Parents should be calm and handle wisely the situation. Parents should set your mind at rest and they are safe at home. Parents should assure their children about safety and should feel negative energy. It has been found that although children are not affected by COVID 19 infection but psychological impacts have been seen [4].

\section{Impact of COVID 19 on patients [5]}

For the period of the precedent few weeks we have accomplished a noteworthy raise in phone contacts and visits from patients or near ones expressing misery and fright. Patients who have mental health conditions and medical comorbidities, such as CVS disease, coupled with deterioration of symptoms, in case of angina.

\section{Impact of COVID 19 on elders and people and disabilities [4]}

Elderly people have more chances of COVID-19 pandemic due to weaker immune system and are being separated from their families and acquaintances due to their hectic schedules. So people with age above 60 are more prone to COVID 19 and due to separation can have sweeping negative effects on the mental health of disabled and the elderly population. Isolation at home can cause distress, anxiety and stimulate a shocking situation for them. However just a telephonic call can help to cheer up elderly people.

Physician trainee burnouts A short online survey conducted [6]

COVID-19 pandemic has put huge twist on frontline healthcare workers. It was found physician trainees were experiencing significant challenges during the pandemic. A total number of 1375 physician trainees at an academic medical center were called up to play a part in an online survey. And it was found that only 393 trainees completed the survey. On winding up concluding it was found that the exposed group had a higher prevalence of stress and burnout. While female trainees and unmarried were more likely 
to be depressed. So wellness programme should be conducted to develop mental health.

\section{Impact on health care workers}

Health-care workers have sustained to offer concern for patients in spite of overtiredness and personal risk of infection. Sadly, health-care workers have come across with also faced many sources of stress, anxiety and long shifts. Hospital-based health professionals are working in bulky and tight personal protective equipment (PPE). Health-care workers had to show concern for colleagues, console dying patients. Health-care workers are identified to be at risk for depression, anxiety, insomnia, moral distress, burnout, and post-traumatic stress disorder [7]. A detailed psychological crisis intervention plan should be developed to deal with the secondary mental health problems involved in the COVID-19 pandemic, urgent psychological crisis intervention model (PCIM) should be developed and implemented. This PCIM integrate teams of psychiatrists, mental health practitioners, physicians, psychologists and social workers to deliver early psychological intervention to patients, medical staff and families [8].

Mitigating psychological effects of COVID 19 on health care workers [9]

Greatest risk of psychologic distress is seen among care providers who treat patients with COVID 19. A survey is being carried in among 1257 nurses and physicians who were concerned with patients in China found that these care providers had significantly more depression, insomnia, distress and anxiety than providers who have no direct contact with patients. Another observational study was carried out among 180 health care workers contributing direct care for patients with COVID-19 found ample levels of anxiety and stress that adversely influenced sleep quality and selfefficacy of the individuals. But a strong social support network provides less stress.

\section{Physiological first aid [10]}

Psychological first aid (PFA) is defined as a 'humane, supportive response to a fellow human being who is suffering and who may need support' (World Health Organization, War Trauma Foundation and World Vision International, 2011). The PFA model provides immediate help and support to individuals during crisis. Fox., et al. in 2012 narrated that despite the limitations, key outcomes of PFA are safety feeling calmness and hopeful, thus effective in helping long-term recovery as compared to folks lacking professional mental health training. PFA is simple and straightforward and hence privacy should be maintained open if the person wishes.

\section{Studies proving COVID 19 and mental stress}

Selvaraj P., et al. in 2020 [11] conducted a study in which they assessed the occurrence of depression, psychological distress, stress, insomnia and anxiety practiced by the Indian healthcare workers. A cross-sectional study was carried on among 777 doctors from corona wards from and questionnaire was offered. Hence it was found that early screening which targets the medical work force and the implementation of psychological interventions is crucial for health-care system.

Benjamin Y.Q. Tan., et al. in 2020 [12] examined the psychological distress, anxiety and depression experienced by health care workers in Singapore and further compared between medically and non-medically trained hospital personnel. A self administered questionnaire was prepared and health care workers from 2 major tertiary institutions in Singapore caring for corona patients were invited to participate. Thus, winding up it was found that nonmedical health care personnel are more at risk for psychological distress So early psychological interventions are must.

Shaukat N., et al. in 2020 [13] reviewed and summarized the impacts of COVID-19 pandemic on health-care workers (HCWs). Arksey 0'Malley framework was used to conduct a scoping review. A systematic literature search was done and two data base were used i.e. Google Scholar and Pubmed. We found 154 articles. And on conclusion it was found that the frontline healthcare workers are at risk of physical and mental consequences.

Sritharan J., et al. in 2020 [14] reviewed existing literature and assessed hence understanding the mental health issues that come out during a pandemics. Of the 203 unique articles reviewed, 16 articles were included in this study. On concluding it was found that the impact on frontline workers and those distress from mental health should be prioritized during this pandemic.

Kaur A., et al. in 2021 [15] conducted a meta-analysis and systematic review to a highpoint with current literature and established a data relating the mortality of (COVID-19) in patients with asthma and those deviod of asthma. The databases were explored for studies associating the clinical results with and without asth- 
matic patients diagnosed with COVID-19. Hence it is concluded that asthma may not upsurge the mortality of COVID-19.

\section{Conclusion}

This review provides a brief description of the current COVID-19 situation and illustrates the mental impact on public and healthcare professionals and its Safety measures such as prevention, precautions and vaccination are some of the methods to be taken care of along with special emphasis should be given on Psychological first aid (PFA) and psychological counseling.

\section{Acknowledgement}

This paper would not have been possible without the extraordinary support of my family and my co-authors. I would also like to show my gratitude to my wonderful parents, brothers, family, friends and especially Kimiko Mori-Chhapyanand Dr Harnoor Singh Sandhu.

\section{Bibliography}

1. Kaur A., et al. "Corona Virus "Ghastly pandemic" - A comprehensive literature". Annals of R.S.C.B 25.6 (2021): 404-409.

2. Braquehais MD., et al. "The impact of the COVID-19 pandemic on the mental health of healthcare professionals". QJM: An International Journal of Medicine 113.9 (2020): 613-617.

3. Wiersinga W J., et al. "Pathophysiology, Transmission, Diagnosis, and Treatment of Coronavirus Disease 2019 (COVID-19): A Review". JAMA 324.8 (2020): 782-793.

4. Javed B., et al. "The coronavirus (COVID-19) pandemic's impact on mental health". International Journal of Health Planning and Management 10 (2020): 1-40.

5. Tsamakis K., et al. "COVID-19 pandemic and its impact on mental health of healthcare professionals". Experimental and Therapeutic Medicine 19.6 (2020): 3451-3453.

6. Kannampallil TG., et al. "Exposure to COVID-19 patients increases physician trainee stress and burnout". PLOS ONE 15.8 (2020): e0237301.

7. Mehta S., et al. "COVID 19 a heavy toll on health care workers". The Lancet Respiratory Medicine 9.3 (2021): 226-228.

8. Rana W., et al. "Mental health of medical workers in Pakistan during the pandemic COVID-19 outbreak". Asian Journal of
Psychiatry 51 (2020).

9. Peter E Wu., et al. "Mitigating the psychological effects of COVID-19 on health care workers". CMAJ 192.17 (2020) E459E460.

10. Minihan E., et al. "COVID-19, mental health and psychological first aid". Irish Journal of Psychological Medicine 37.4 (2020): $259-263$.

11. Selvaraj P., et al. "Psychological impact of COVID-19 pandemic on health-care professionals in India - A multicentric crosssectional study". Indian Journal of Medical Sciences (2020).

12. Benjamin YQ Tan., et al. "Psychological Impact of the COVID-19 Pandemic on Health Care Workers in Singapore". Annals of Internal Medicine (2020): M20-1083.

13. Shaukat N., et al. "Physical and mental health impacts of COVID-19 on healthcare workers: a scoping review". International Journal of Emergency Medicine 13.1 (2020): 40.

14. Sritharan Jeavana and Sritharan Ashvinie. "Emerging Mental Health Issues from the Novel Coronavirus (COVID-19) Pandemic". In: Journal of Health and Medical Sciences 3.2 (2020): 157-162.

15. Kaur A., et al. "A Systematic Review and Meta-Analysis on role of Asthma on Mortality rate of Patients with COVID-19". European Journal of Molecular and Clinical Medicine 2 (2021): 330-335.

\section{Volume 5 Issue 8 August 2021}

(C) All rights are reserved by Avineet Kaur., et al. 